\title{
Vehicle Motion Planning for the Visually Challenged People Using Ant Colony Optimization
}

\author{
Dr. S. Prasanna \\ Assistant Professor, Department of Computer Science \\ Shri Shankarlal Sundarbai Shasun Jain College for Women, T. Nagar, Chennai 600017 \\ Email: sprasannaganesan@gmail.com \\ Dr. T. Indumathi \\ Assistant Professor, Department of Computer Application \\ Mar Gregorios College, Mugappair West, Chennai - 600037 \\ Email id:indumathit1979@gmail.com
}

\begin{abstract}
This paper presents a novel proposal to solve the problem of obstacle detection and path planning for visually challenged people based on simple Ant Colony Optimization Meta-heuristic (SACO-MH). The mission of the path planning problem is to enable the vehicle to move from the starting point to the target point while satisfying certain constraints. Constraint conditions are: not a collision with known or unknown obstacles, away from the obstacle as far as possible, determines the shortest path, shortest time and so on. Obstacle detection is made with the help of sensor technology and it is intimated to the user with the help of a smart watch. A voice based navigation system guides the user.
\end{abstract}

Keywords - Particle Swarm Optimization (PSO), Genetic Algorithm (GA), Tabu Search, Simulated Annealing (SA), Reactive Search Optimization (RSO).

\section{INTRODUCTION}

The blinds are a special group of life, due to the limited vision or no vision, face many inconveniences in their lives. This invention belongs to a technical field of automated system and a controller with a voice guided navigation system which can be used by the visually challenged people. These people often walk with crutches to find the obstacles but they are conventional and not with the intelligence. Since the smart technology is growing, it is important to know how to use the smart technology to improve the lives of visually challenged people. The technical solution for this invention works as: The user is provided with a vehicle which consists of a single controller to monitor the directions and movements of the vehicle. Now, to detect the movable and immovable obstacles on the path, the vehicle is built with sensors and navigation systems. And, the user is given a smart watch to indicate the obstacles by some vibrations or alarm kind of things. The smart watch and the navigation systems are interfaced with the sensors. While the sensor detects the obstacles, it gives the signals to both the smart watch and the navigator. The smart watch will vibrate to indicate the obstacle to the 
user and the navigator instructs the user to move in some other direction to reach the target point. So, to detect the obstacles a sensor called LIDAR is used, that measures distance to a target by illuminating that target with a pulsed laser light, and measuring the reflected pulses with a sensor. The technology is used for control and navigation for some autonomous cars. Lidar sometimes is called laser scanning and 3D scanning, with terrestrial, airborne, and mobile applications. Once the obstacle is detected, the Ant Colony Optimization (ACO) technique is used o find the shortest path in shortest time. The main objective of this paper is to explore the efficient algorithms to solve the problem of dependency for the visually challenged ones.

1.1 Particle Swarm Optimization (PSO): Particle Swarm Optimization (PSO) is a computational method that optimizes a problem by iteratively trying to improve a candidate solution with regard to a given measure of quality. PSO optimizes a problem by having a population of candidate solutions, with dubbed particles, and moving these particles around in the search-space according to simple mathematical formulae over the particle's position and velocity. Each particle's movement is influenced by its local best known position and is also guided toward the best known positions in the search-space, which are updated as better positions are found by other particles. This is expected to move the swarm toward the best solutions. PSO is a meta heuristic as it makes few or no assumptions about the problem being optimized and can search very large spaces of candidate solutions. However, meta heuristics such as PSO do not guarantee an optimal solution is ever found. More specifically, PSO does not use the gradient of the problem being optimized, which means PSO does not require that the optimization problem be differentiable as is required by classic optimization methods such as gradient descent and Quasi-Newton methods. PSO can therefore also be used on optimization problems that are partially irregular, noisy, change over time.

\section{Genetic Algorithm (GA)}

A genetic algorithm (GA) is a search heuristic that mimics the process of natural evolution. This heuristic is routinely used to generate useful solutions to optimization and search problems. Genetic algorithms belong to the larger class of evolutionary algorithms (EA), which generate solutions to optimization problems using techniques inspired by natural evolution, such as inheritance, mutation, selection, and crossover. In a genetic algorithm, a population of strings called chromosomes or the genotype of the genome, which encodes candidate solutions called individuals, creatures, or phenotypes to an optimization problem, evolves toward better solutions. Traditionally, solutions are represented in binary as strings of $0 \mathrm{~s}$ and $1 \mathrm{~s}$, but other encodings are also possible. The evolution usually starts from a population of randomly generated individuals and happens in generations. In each generation, the fitness of every individual in the population is evaluated, multiple individuals are stochastically selected from the current population (based on their fitness), and modified (recombined and possibly randomly mutated) to form a new population. The new population is then used in the next iteration of the algorithm. Commonly, the algorithm terminates when either a maximum number of generations has been produced, or a satisfactory fitness level has been reached for the population. If the algorithm has terminated due to a maximum number of generations, a satisfactory solution may or may not have been reached. 
2.1 Tabu Search: Tabu search is a local search method used for mathematical optimization. Local searches take a potential solution to a problem and check its immediate neighbors in the hope of finding an improved solution. Local search methods have a tendency to become stuck in suboptimal regions or on plateaus where many solutions are equally fit. Tabu search enhances the performance of these techniques by using memory structures that describe the visited solutions or user-provided sets of rule. If a potential solution has been previously visited within a certain short-term period or if it has violated a rule, it is marked as "taboo" so that the algorithm does not consider that possibility repeatedly.

2.2 Simulated Annealing (SA): Simulated annealing (SA) is a generic probabilistic meta heuristic for the global optimization problem of locating a good approximation to the global optimum of a given function in a large search space. It is often used when the search space is discrete. For certain problems, simulated annealing may be more efficient than exhaustive enumeration - provided that the goal is merely to find an acceptably good solution in a fixed amount of time, rather than the best possible solution. The name and inspiration come from annealing in metallurgy, a technique involving heating and controlled cooling of a material to increase the size of its crystals and reduce their defects. The heat causes the atoms to become unstuck from their initial positions (a local minimum of the internal energy) and wander randomly through states of higher energy; the slow cooling gives them more chances of finding configurations with lower internal energy than the initial one. By analogy with this physical process, each step of the SA algorithm attempts to replace the current solution by a random solution (chosen according to a candidate distribution, often constructed to sample from solutions near the current solution). The new solution may then be accepted with a probability that depends both on the difference between the corresponding function values and also on a global parameter T (called the temperature), that is gradually decreased during the process. The dependency is such that the choice between the previous and current solution is almost random when $\mathrm{T}$ is large, but increasingly selects the better or "downhill" solution (for a minimization problem) as T goes to zero. The allowance for "uphill" moves potentially saves the method from becoming stuck at local optima-which are the bane of greedier methods.

2.3 Reactive Search Optimization (RSO): Reactive Search Optimization (RSO) defines local-search heuristics based on machine learning, a family of optimization algorithms based on the local search techniques. It refers to a class of heuristics that automatically adjust their working parameters during the optimization phase. Reactive Search Optimization (RSO), like all local search techniques, is applied to the problem of finding the optimal configuration of a system; such configuration is usually composed of continuously or discretely varying parameters, while the optimality criterion is a numerical value associated to each configuration. In most cases, an optimization problem can be reduced to finding the (global) minimum of a function whose arguments are the configuration parameters, seen as free variables in the function's domain space. Reactive Search Optimization advocates the integration of subsymbolic machine learning techniques into search heuristics for solving complex optimization problems. The word reactive hints at a ready response to events during the search through an 
internal feedback loop for online self-tuning and dynamic adaptation. In Reactive Search the past history of the search and the knowledge accumulated while moving in the configuration space is used for self-adaptation in an autonomic manner: the algorithm maintains the internal flexibility needed to address different situations during the search, but the adaptation is automated, and executed while the algorithm runs on a single instance and reflects on its past experience.

2.4 Ant colony algorithms: In the natural world, ants (initially) wander randomly, and upon finding food return to their colony while laying down pheromone trails. If other ants find such a path, they are likely not to keep traveling at random, but to instead follow the trail, returning and reinforcing it if they eventually find food. Below figure 1 illustrates the same. Over time, however, the pheromone trail starts to evaporate, thus reducing its attractive strength. The more time it takes for an ant to travel down the path and back again, the more time the pheromones have to evaporate. A short path, by comparison, gets marched over more frequently, and thus the pheromone density becomes higher on shorter paths than longer ones. Pheromone evaporation also has the advantage of avoiding the convergence to a locally optimal solution. If there were no evaporation at all, the paths chosen by the first ants would tend to be excessively attractive to the following ones. In that case, the exploration of the solution space would be constrained.

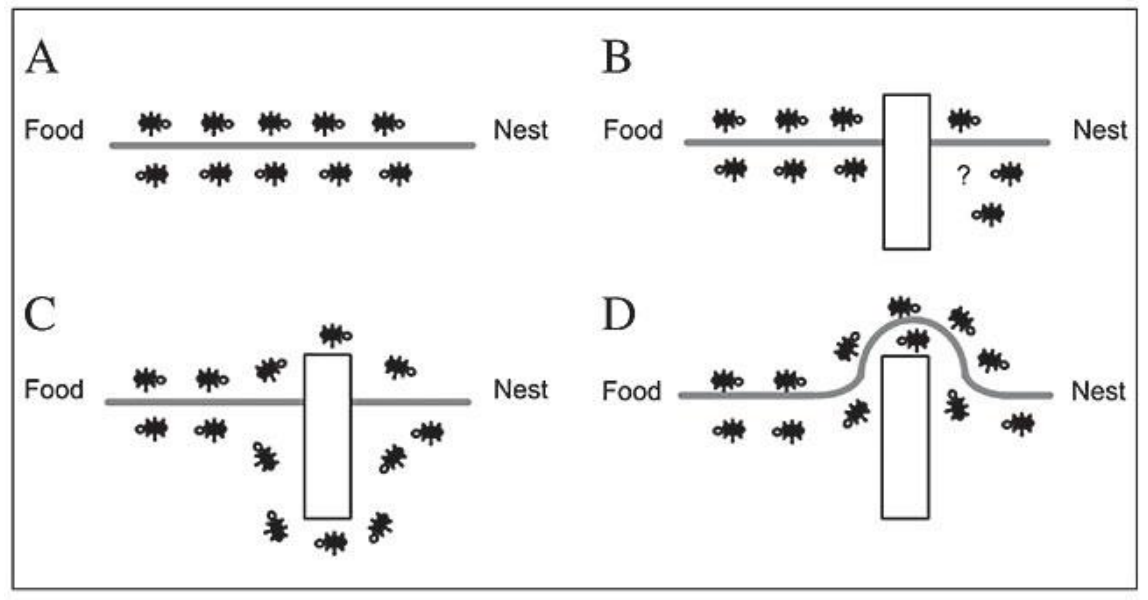

Fig 1: A: Ants in a Pheromone train between nest and food. B: an obstacle interrupts the train. $C$ : ants find two paths to go around the obstacle D: a new pheromone trail is formed along the shorter path.

2.5 Lidar - LIght Detection And Ranging (also called LIDAR, LiDAR, and LADAR) is a surveying method that measures distance to a target by illuminating that target with a pulsed laser light, and measuring the reflected pulses with a sensor.The technology is used for control and navigation for some autonomous cars. Lidar sometimes is called laser scanning and 3D scanning, with terrestrial, airborne, and mobile applications.[1] 
2.6 Vibrator synced smart watches - It's a creative idea that takes advantage of the latest advancement in technology and applies it in a meaningful way to our product. The procedure simulates the decision-making processes of ant colonies as they forage for food and is similar to other adaptive learning and artificial intelligence techniques such as Tabu Search, Simulated Annealing and Genetic Algorithms. Modifications are made to the ACO algorithm used to solve the traditional traveling salesman problem in order to allow the search of the multiple routes of the VRP. Experimentation shows that the algorithm is successful in finding solutions within $1 \%$ of known optimal solutions and the use of multiple ant colonies is found to provide a comparatively competitive solution technique especially for larger problems. Additionally, the size of the candidate lists used within the algorithm is a significant factor in finding improved solutions, and the computational times for the algorithm compare favorably with other solution methods.[2]

\section{Overcoming the Problem of ACO}

Various approaches to overcome the problem of ACO i.e. mitigating stagnation which include:evaporation, aging and pheromone smoothing [3]. The Approaches to alleviate stagnation is pheromone control. Pheromone control adopts several approaches to reduce the influence from past experience and encourage the exploration of new paths that are non-optimal.

3.1 Evaporation: To reduce the effect of past experience, an approach called evaporation is used in conjunction in optimal path from being excessively high and preventing ants from exploring the other paths. In each iteration, the pheromone value Tij in all edges are decremented by a factor $\mathrm{p}$ such that Tij $\leftarrow$ Tij $(1-\mathrm{p})$

3.2 Aging: A past experience can also be reduced by controlling the amount of pheromone deposited for each ant according to its age. This approach is known as aging. In aging, an ant deposits lesser and lesser amount of pheromone as it moves from one obstacle to other obstacle. Aging is based on the rationale that -old ants are less successful in locating the optimal paths since they take longer time to reach their destination. Both aging and evaporation encourage discoveries of new paths that are previously non-optimal

3.3 Limiting and smoothing pheromone: Limiting the amount of pheromone in every path, by placing an upper bound on the amount of pheromone for every edge $(\mathrm{i}, \mathrm{j})$, the preference of an ant for optimal path is reduced. This approach prevents the situation of generating a dominant path. A variation of such an approach is called pheromone smoothing.

\section{CONCLUSION}

Ant colony algorithms have been widely used to solve various optimization problems in different fields, especially in the field of engineering design. Ant colony algorithm is an intelligent algorithm with positive feedback mechanism. The main content includes the construction of path and the update of pheromone. After an in-depth study of the ant colony algorithm, an ant colony algorithm with punitive measures is proposed. The salient aspect of 
this punitive measure is that at the end of each generation, the ant finds a poorer path pheromone volatilization rate, thereby reducing the re-exploration of this path and increasing the opportunity to explore the unknown. In this paper we presented a novel proposal to solve the problem of obstacle detection and path planning for visually challenged people based on simple Ant Colony Optimization Meta-heuristic (SACO-MH). This algorithm performs better in dealing with unmanned vehicle path planning.

\section{REFERENCES:}

[1] Lee, Sang-Mook; Joon Im, Jeong; Lee, Bo-Hee; Leonessa, Alexander; Kurdila, Andrew. "A real time grid-map generation and object classification for ground based $3 D$ lidar data using image analysis techniques". Image Processing (ICIP), 2010 17th IEEE International Conference on image processing. ISSN 1522-4880.

[2] Shim, H.M., Lee, J.S., Lee, E.H., Hong, S.H.: A Study on the Sound-Imaging Algorithm of Obstacle Information for the Visually Impaired. In: ITC-CSCC 2002, pp. 389-39 (2002)

[3] M.A. Porta Garcia, Oscar Montiel,Oscar Castillo, Robert Sepulveda ,Patricia Melin, "Path planning of autonomous mobile robot navigation with ant colony optimization and fuzzy cost function evaluation”, Applied Soft Computing, Volume 9, Issue 3 ,pp.1102-1110, June 30, 2009. 